\title{
MICROBICIDES: A NEW APPROACH TO PREVENTING HIV AND OTHER SEXUALLY TRANSMITTED INFECTIONS
}

MICROBICIDE

(Virucide). An anti-infective medication formulated for topical self-administration before intercourse to protect against HIV and other sexually transmitted pathogens.

PATHOGEN

A microorganism that causes disease.
International Family Health, Cityside House, 40 Adler Street, London E1 1EE, UK. e-mail: alan.stoneX@virgin.net doi:10.1038/nrd959

\section{Alan Stone}

Many chemical agents can block human immunodeficiency virus (HIV) infection in the laboratory. Scientists are investigating which of these, used intravaginally by women, will safely prevent the sexual transmission of HIV in humans. Several such products - microbicides will soon be tested in populations at high risk for HIV, and others are waiting in the wings. Microbicides will provide a low-cost method, controlled by women, for protection against HIV, other sexually transmitted pathogens and unwanted pregnancy, and will therefore have global public-health benefits.

Vaginal medications containing the spermicidal surfactant nonoxynol-9 have been used for more than 50 years to reduce the risk of unwanted pregnancy. The demonstration that nonoxynol-9 not only attacks sperm but can also destroy the human immunodeficiency virus $(\mathrm{HIV})^{1,2}$ provided the initial stimulus to search for products that, when used vaginally, would prevent the sexual transmission of this deadly virus. Initially termed 'virucides', the name now commonly accepted for such products is 'MICROBICIDES', reflecting the intention that they should protect not only against viruses, such as HIV and genital herpes, but also against common bacterial infections, such as gonorrhoea and chlamydia.

The basic concept is simple. Anti-infective chemicals, selected from the great variety of substances that are known to block HIV and other sexually transmitted PATHOGENS in the laboratory, are formulated to create products suitable for insertion into the vagina before intercourse. These can be in the form of gels, creams, foams, impregnated sponges, suppositories or films. The chemical and physical actions of the product will protect the uninfected person, man or woman, from infectious agents that might be present in the genital secretions of his or her sexual partner. Microbicides will also protect HIV-infected people from other sexually transmitted infections (STIs), including possibly drug-resistant or more virulent HIV strains. This will be particularly important in immunocompromised individuals, who are especially vulnerable to infections. Research is also under way on microbicides for use in rectal sex.

Although this might sound straightforward, the process of developing and evaluating microbicides is highly complex. As well as providing effective protection, they must also be safe to use, chemically and physically stable, compatible with latex and other materials used in barrier devices, and affordable and acceptable to the end-user.

\section{The need for microbicides}

Why do we need to develop microbicides given that efforts to produce anti-HIV vaccines are under way and, in addition, the condom is known to offer good protection against HIV and other STIs? Regrettably, the development of an AIDS vaccine - which will ultimately have a crucial role in the war against the HIV pandemic - is proving to be a great deal more technically challenging than many had expected. Vaccines suitable for largescale administration over diverse geographic regions might be many years away. Furthermore, condoms are effective against these infections only if they are used consistently and correctly ${ }^{3,4}$, and this is seldom the case. Unprotected sex remains widespread, as can be judged from the continuing growth of the global HIV epidemic (4.3 million new adult cases of HIV during 
EPITHELIUM

The tissue that covers the surface of the body and lines hollow structures, including the male and female genital tracts and the rectum. Its structure varies depending on location and role.

ULCER

A lesion in the epithelium that extends through to the underlying connective tissue.
2001, 1.8 million of them in women ${ }^{5}$ ) and from the annual burden of around 340 million cases of other $\mathrm{STIs}^{6}$. Intensive campaigns to promote condoms remain as important as ever, and there have been some successes; most notably in Thailand and Uganda. But the fact remains that they are not popular, particularly with men, and in many of the societies in which HIV is prevalent, women lack the power to negotiate their use.

By contrast, microbicides will provide a user-friendly technology that will widen the range of protective options and, importantly, will be under the control of women. Several studies predict that they will be widely acceptable to both men and women ${ }^{7-9}$. Unlike condoms, microbicides will not create a physical barrier to intimate contact, and women will be able to apply them a considerable time before intercourse takes place. Ideally, they will be colourless, odourless and tasteless, and non-messy to use. Moreover, the use of a microbicide need not necessarily prevent conception, as the intention is to develop both contraceptive and noncontraceptive products.

\section{Studies with nonoxynol-9}

The need for microbicides is urgent. A fast-track approach is to investigate products that are already licensed for vaginal use, such as the spermicidal contraceptives that are now on the market, and find out whether they will protect individuals in a population at high risk of HIV infection. Nonoxynol-9 is a surfactant that works as a spermicide by disaggregating the lipid membranes of spermatozoa. It has a similar effect on the lipid membranes of HIV, the genital herpes virus and sexually transmitted cellular pathogens, such as Chlamydia trachomatis and Neisseria gonorrhoeae (FIG. 1a).

a

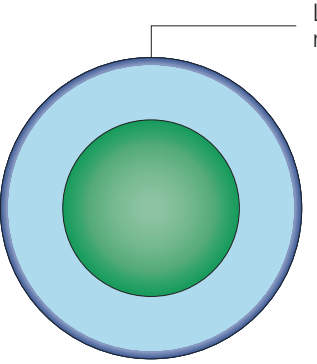

Lipid
membrane
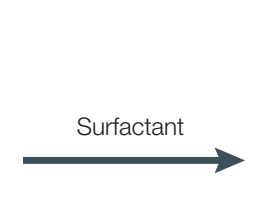

Fragmented
membrane

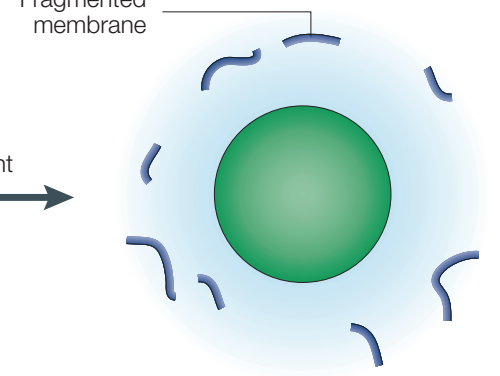

b
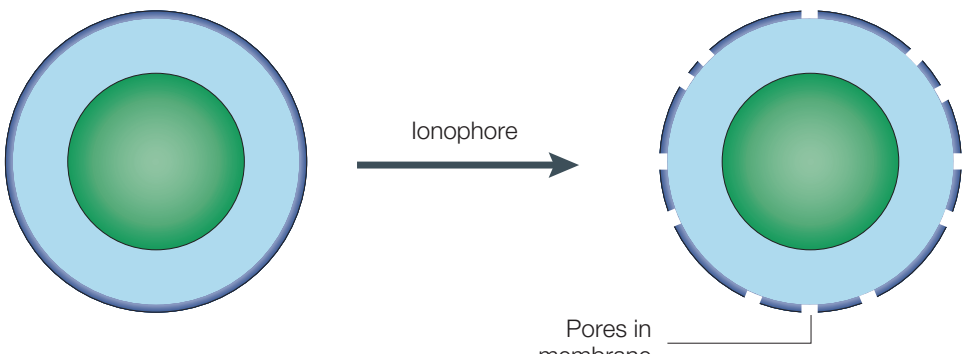

membrane

Figure 1 | Some microbicides work by damaging the lipid membrane of the pathogen. a | Surfactants, such as nonoxynol-9 and C31G, disaggregate the membranes of cells and enveloped viruses. b | Certain antibiotic peptides, such as gramicidin and defensins, act as ionophores and create holes in the membrane.
The first large-scale (Phase III) clinical trial to evaluate nonoxynol-9 involved female sex workers in Nairobi ${ }^{10}$. To be statistically significant, such trials typically require several thousand female volunteers who are willing to be randomized to use either the microbicide or a placebo, and who are also willing to undergo regular testing for HIV and other STIs over many months.

Phase III trials are usually designed to provide information on the long-term safety and acceptability of a product, as well as its effectiveness. However, a trial on this scale, with its logistical complexities and exacting ethical requirements ${ }^{11}$, is justified only if the product has previously been shown to be safe and acceptable in smaller trials. A Phase I trial provides initial data in perhaps 10-20 healthy women at low risk of infection. This is followed by a Phase II trial to confirm the findings over a longer period in perhaps 200-300 women — including some HIV-positive women - preferably in a population similar to that in which it is intended to carry out the Phase III trial. Safety end points include the absence of systemic toxicity and local adverse effects on the EPITHELIUM that lines the vagina and cervix, and minimal disturbance of the bacterial flora normally resident in the healthy vagina. Penile safety studies are also necessary. Detailed information about clinical studies in microbicide development is available in a document prepared for the International Working Group on Microbicides ${ }^{11}$.

In the Nairobi trial, the product being tested was a commercially available contraceptive sponge impregnated with 1,000 mg of nonoxynol-9. The outcome was disappointing: the product did not protect against HIV, gonorrhoea or chlamydia, and seemed to be responsible for genital uLCERS in some of the women ${ }^{10}$. Several subsequent Phase III trials of different nonoxynol-9 formulations also failed to show any benefit. These included a study in the Cameroon of a vaginal contraceptive film incorporating $70 \mathrm{mg}$ of nonoxynol-9 (REF. 12) and a trial of a contraceptive gel (known as COL-1492) containing 52.5 $\mathrm{mg}$ of the substance, which was carried out in several African sites ${ }^{13}$. In the latter trial, use of the surfactant was associated with a significant increase in the incidence of HIV infection. This was particularly pronounced among the women who were more frequent users of the product (mean use $>3.5$ times a day), and in the same group, there was a higher incidence of genital ulcers than in the equivalent placebo group. In neither trial was there an effect on gonorrhoea or chlamydia infection rates. It is unclear why the earlier safety studies with COL-1492 failed to show an excess of ulcers ${ }^{14}$, but this was probably because of their short duration and insufficient power to detect an effect of the size seen in Phase III studies. In a further study carried out in the Cameroon, a different gel containing $100 \mathrm{mg}$ nonoxynol-9 also failed to protect women against the two bacterial infections (HIV was not a primary end point in this trial $)^{15}$.

After the initial announcement of the COL-1492 result in 2000, several national and international bodies issued statements advising on its implications for the use of nonoxynol-9 in both stand-alone products and condom coatings. In June 2002, the World Health Organization released a report containing the 


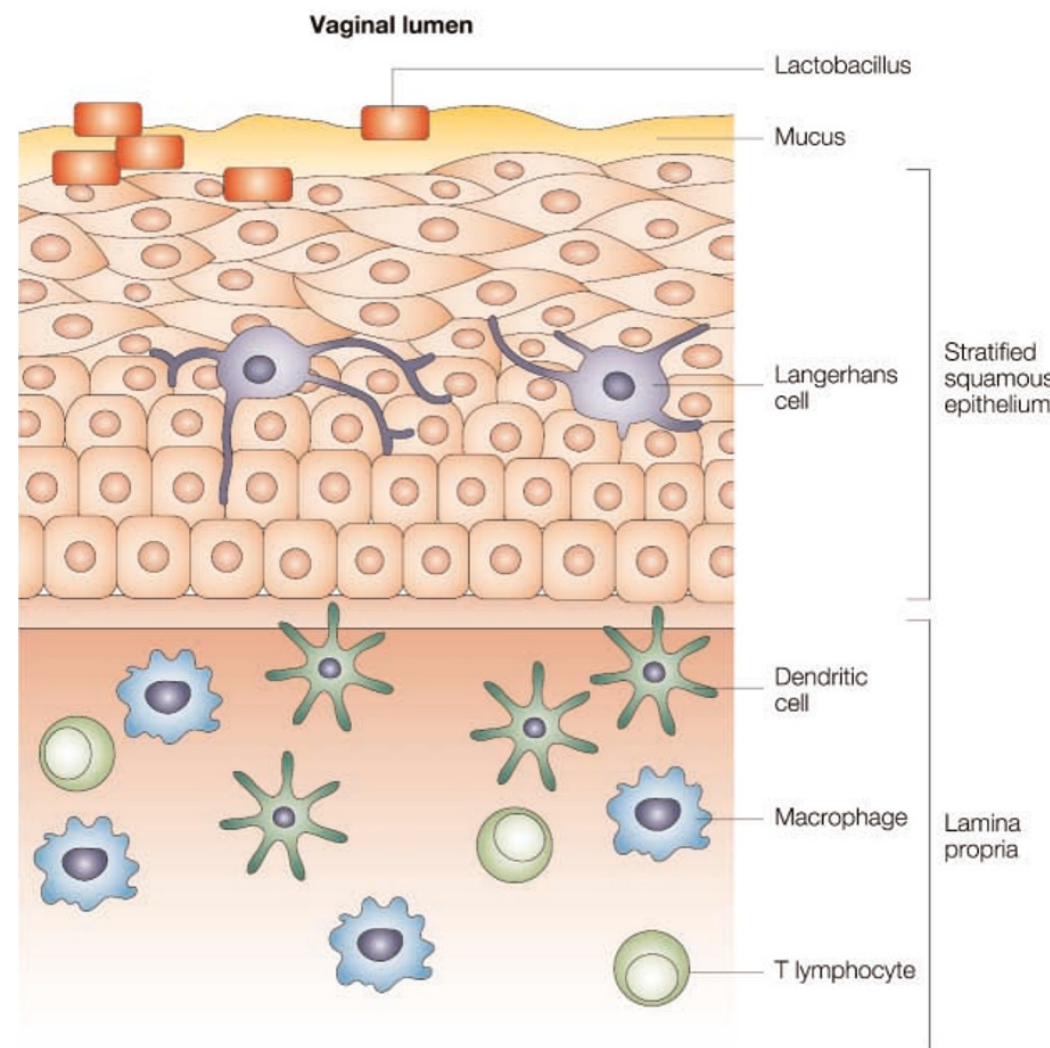

Figure 2 | Structure of the human vaginal epithelium. The multilayered epithelium and the underlying connective tissue contain several types of potential target cell for HIV.

LYMPHOID CELLS

Types of immune cell, including macrophages, lymphocytes, Langerhans cells and dendritic cells, that have specific roles in the diverse components of the immune response to foreign antigens.

GLYCOPROTEIN

A protein combined with a carbohydrate, such as mannose or galactose.

CHEMOKINES

A group of small proteins involved in intracellula signalling - a subgroup of the cytokines.

EXPLANT

Living tissue excised from the body and maintained in culture medium. recommendations of a meeting of experts who had concluded, after careful scrutiny of all the available evidence on the potential benefits and hazards of nonoxynol-9, that this substance should not be used as a microbicide (although it remains a contraceptive option for women at low risk of HIV $)^{16}$. The report stresses that alternative microbicides, which are safe and effective, need to be developed as a matter of urgency.

There is insufficient information to be able to offer definitive guidance about the value of other surfactants as microbicides, although research is continuing with a number of them. For example, C31G, an equimolar mixture of the amphoteric surfactants cetyl betaine and myristamine oxide, has successfully completed a Phase I safety study (A.-M. Corner, personal communication).

\section{Mechanism of HIV transmission}

To understand why nonoxynol-9 failed to protect against HIV in the above trials, and why there is considerable optimism about the eventual success of some of the alternative microbicides that are under investigation at present, we need to consider the biological mechanism by which HIV is transmitted sexually. Potential sources of transmissible HIV are free virus particles and infected LYMPHOID CELLS in semen, in cervicovaginal secretions and in blood or other fluids present as a result of physical trauma or genital infections. The first steps in HIV infection normally involve the attachment of the virus, through the gp120 GLYCOPROTEIN on its outer membrane, to its primary receptor, $\mathrm{CD} 4$, on the surface of the host cell. This is followed by an interaction between a specific gp120 domain and a cellular co-receptor, which triggers a conformational change in the viral envelope that leads to fusion of the viral and host-cell membranes and entry of the viral genome into the host cell ${ }^{17}$. Two CHEMOKINE receptors on the cell surface CCR5 and CXCR4 - serve as co-receptors for HIV, and different HIV strains tend to have a strong preference for one or the other.

The epithelium that lines the vagina and the external surface of the cervix is multilayered, and is relatively strong and durable. Beneath this is a layer of connective tissue - the lamina propria. Within these structures are several classes of lymphoid cell, including dendritic cells, macrophages, T lymphocytes and Langerhans cells (FIG. 2). Much has been written about the role of these tissues and cells in the transmission of HIV from males to females, and some aspects are controversial. (The detailed mechanism by which penile tissue becomes infected is also unclear, although there are likely to be some similarities.) A considerable body of evidence has been derived from experiments on EXPLANTS of human vaginal and cervical tissues infected with HIV in vitro, and from studies in macaque monkeys infected vaginally with the simian immunodeficiency virus (SIV). Most of the findings support the view that the primary initial sites of HIV infection are lymphoid cells in the lamina propria - dendritic cells and macrophages in particular, but possibly also T lymphocytes - that have both CD4 and the coreceptors for the virus ${ }^{18-20}$. The Langerhans cells in the genital epithelium (in contrast to those in human skin) do not seem to be as readily infectable by the incoming virus, probably because, although they express CD4, they express little of the co-receptors ${ }^{18}$. However, the possibility that they have a role in the infection process cannot be ruled out.

Infected dendritic cells can migrate to the local lymph nodes, where extensive HIV replication takes place, leading to generalized systemic infection. HIV particles can also attach to the surface of dendritic cells, without infecting them, through an interaction between mannose-rich residues in gp120 and specific lectins in the cell membrane, including one known as DC-SIGN. The significance of this finding is not yet clear, but it is possible that this is an important route by which non-replicating, but infectious, HIV could be carried to the lymph nodes and be presented to susceptible cells ${ }^{21}$. If so, it provides another potential target for microbicide intervention.

The non-lymphoid squamous cells, which comprise the bulk of the multilayered genital epithelium, have none of the necessary receptors for HIV and - subject to the technical limitations of the assay systems - seem to be resistant to infection ${ }^{18}$. Furthermore, these cells (unlike the cells of the epithelium that lines the gastrointestinal $\operatorname{tract}^{22}$ ) do not allow the virus to migrate through them by passive transcytosis. This raises the question of how HIV - whether the free virus particles or HIV-infected lymphoid cells - manages to pass through the resistant epithelial barrier. Studies in tissue explants have given rise to several proposals ${ }^{18,23-25}$. 
CYTOKINES

Small protein molecules that control the activity of immune cells, produced, for example, as part of the inflammatory response.

\begin{tabular}{|c|c|}
\hline Type of compound & Name \\
\hline \multicolumn{2}{|c|}{ Group A: disrupt or otherwise disable HIV } \\
\hline Surfactants & $\begin{array}{l}\text { Nonoxynol-9 (further investigation unlikely), octoxynol-9, benzalkonium } \\
\text { chloride, menfegol, docosanol, C31G (Savvy), sodium cholate, } \\
\text { polybiguanides, sodium dodecyl sulphate }\end{array}$ \\
\hline Antibiotics & Gramicidin, magainins, defensins, protegrins \\
\hline Acidifying agents & BufferGel, Acidform, Lactobacillus crispatus suppositories \\
\hline Oxidising agents & Chlorhexidine, povidone iodine, hydrogen peroxide/peroxidase gel \\
\hline Antibodies & Anti-HIV 'plantibodies' \\
\hline Long-chain anionic polymers & Cellulose acetate phthalate (also in group B) \\
\hline Reverse-transcriptase inhibitors & UC781 (also in group C) \\
\hline \multicolumn{2}{|c|}{ Group B: block HIV attachment/fusion } \\
\hline Long-chain anionic polymers & $\begin{array}{l}\text { Dextrin-2-sulphate, naphthalene sulphonate polymer (PRO 2000), } \\
\text { carrageenan, polystyrene sulphonate, cellulose sulphate, cellulose acetate } \\
\text { phthalate (also in group A), polymeric sodium dimandelic acid ether (SAMMA) }\end{array}$ \\
\hline Dendrimers & SPL7013 \\
\hline HIV-binding peptides/proteins & Cyanovirin, T-20 \\
\hline Lipid-membrane modifiers & $\beta$-Cyclodextrin \\
\hline Anti-CD4 antibodies & B-12 \\
\hline \multicolumn{2}{|c|}{ Group C: prevent intracellular HIV replication } \\
\hline Reverse-transcriptase inhibitors & Nevirapine, loviride, tenofovir, UC781 (also in group A) \\
\hline \multicolumn{2}{|l|}{ Group D: mechanism unclear } \\
\hline Plant products & Praneem, gossypol, pokeweed antiviral protein \\
\hline
\end{tabular}

One is that Langerhans cells and Tymphocytes in the epithelium might have the capacity to bind the virus and migrate with it to the lamina propria. However, several observations indicate that HIV can reach its subepithelial target cells only if there are physical breaches in epithelial integrity ${ }^{18}$. Clearly, such lesions will make it easier for HIV to infect cells in the lamina propria even if there are other routes through the epithelium. They can result from infection with other pathogens, from physical trauma or, as we have seen, from the use of the surfactant nonoxynol-9.

Surfactant-induced ulcers take several days to heal, so in clinical trials in populations in which sex is frequent, it is perhaps not surprising that nonoxynol-9 showed no net benefit ${ }^{26}$. Phase I studies have shown that nonoxynol-9 can also give rise to localized inflammation ${ }^{27}$, and it is

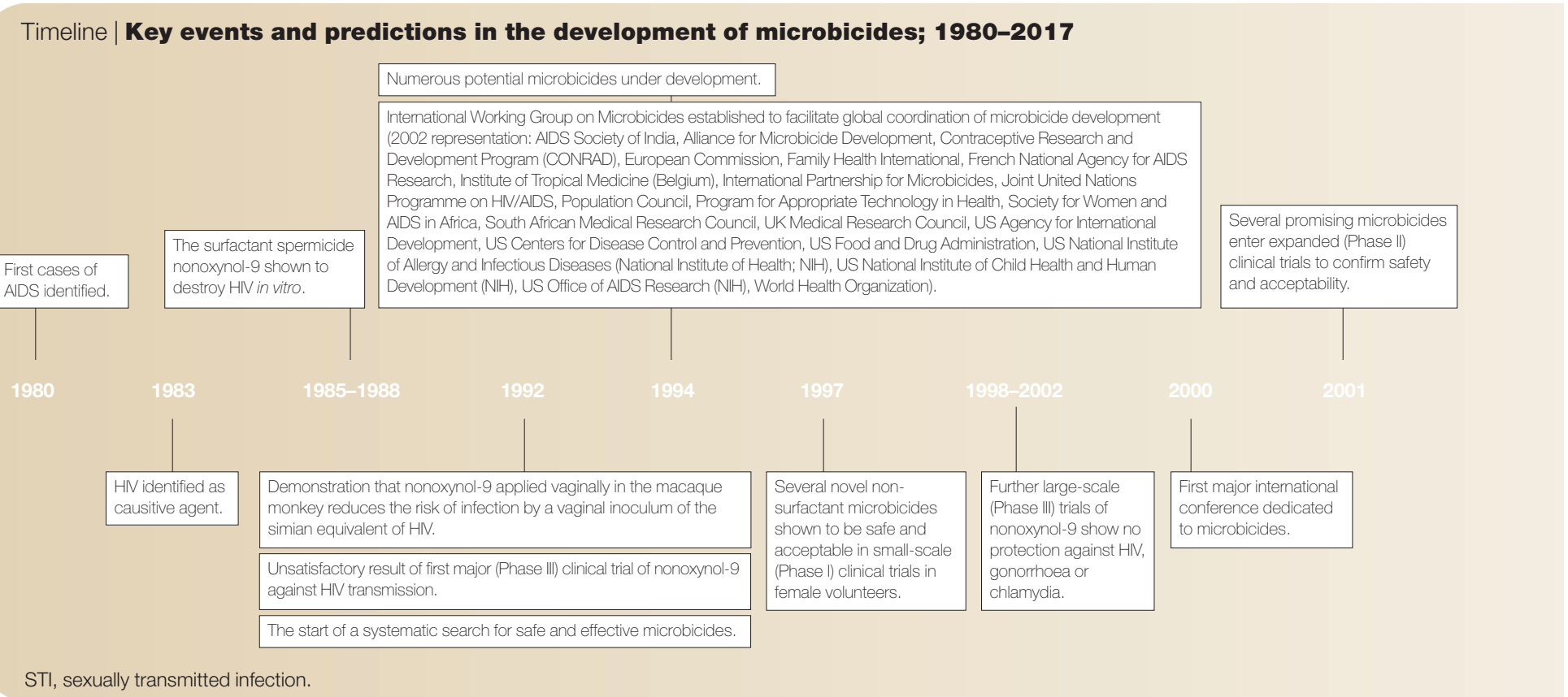


likely that this also enhances the risk of HIV infection; for example, by СүтокіNE activation of potential target cells ${ }^{18}$. Moreover, nonoxynol- 9 adversely affects the lactobacilli that live in the healthy vagina and whose secretion of lactic acid and hydrogen peroxide creates a second line of defence against invading pathogens ${ }^{27-29}$.

\section{Microbicides under investigation}

The problems experienced with nonoxynol-9 have focused attention on microbicides that work by different mechanisms, and which neither damage the vaginal lining nor affect the lactobacilli. Many such agents are now under investigation. To prevent HIV transmission, a microbicide must either inactivate the virus (both free and cell-associated) while it is still in the vaginal lumen, prevent the virus from attaching to and fusing with its host cells (or attaching to DC-SIGN) or prevent the virus from replicating if it should succeed in infecting these cells. TABLE 1 includes examples of substances that have the potential to intervene at one or other of these stages of the HIV infection process. Many are active against a wide range of HIV strains, and most also show some activity against other STI organisms. The latter are responsible for substantial morbidity and mortality. There is also evidence that STIs enhance the susceptibility of an affected person to infection by HIV ${ }^{30}$. So, at the community level, by protecting against these infections, it is likely that microbicides will have an indirect impact on HIV levels as well as a direct impact on the transmission of the virus.

XENOGRAFT

Living tissue from one species grafted into or onto another.

ANIONIC POLYMER

A long-chain molecule composed of linked, negatively charged units.
Preclinical assessment of candidate microbicides. Before such substances can be tested in human subjects, they must undergo a battery of laboratory investigations to establish levels of efficacy and toxicity. Simple in vitro systems for evaluating the efficacy of a microbicide against HIV assess the ability of the agent to prevent viral replication in cultured human lymphoid cells, and its cytotoxic effects can be studied in similar cultures ${ }^{31}$. More sophisticated in vitro systems have also been developed, which are designed to mimic sexual transmission as closely as possible. These include explants of human vaginal or cervical tissue maintained in culture fluid, which can be infected with a range of HIV strains and used to assess microbicide potency under relevant physiological conditions, such as at various $\mathrm{pHs}$ and in the presence of human semen $^{18}$ (R. Shattock, personal communication).

Microbicides can also be tested in various animal systems. Female macaques develop systemic infection if they are given a vaginal inoculum of SIV or SHIV (a genetically engineered hybrid that comprises the viral core of SIV and an HIV outer envelope). Microbicides can be placed in the vagina before the virus to see if they protect against such infection ${ }^{32-36}$. Work is also in progress to develop small-animal models for studying infection/ protection with HIV itself, in immunodeficient mice either reconstituted with human lymphocytes ${ }^{37}$ or bearing human vaginal XENOGRAFTS ${ }^{38}$. The efficacy of microbicides against the human genital herpes virus can be studied both in vitro and also against vaginal infection in a mouse model ${ }^{39-41}$. Activity against $N$. gonorrhoeae and C. trachomatis can be assessed in cultures of these organisms growing, respectively, on agar plates or in suitable human cell cultures.

Thorough toxicological investigations of potential microbicides are imperative to detect any local or systemic effects, or reproductive toxicity. General recommendations for the preclinical assessment of microbicides, including activity, toxicology and formulation, are included in a document produced by the International Working Group on Microbicides ${ }^{42}$ (see TIMELINE), and updated preclinical recommendations will be published shortly.

Anionic polymers. Laboratory tests such as these have resulted in the spotlight being turned on a group of ANIONIC POLYMERs. Unlike nonoxynol-9, they are not cytotoxic, but they are potent inhibitors of HIV infection in vitro. This is illustrated in FIG. 3, which clearly shows that the naphthalene sulphonate polymer PRO 2000 has a far better therapeutic index than the surfactant nonoxynol-9. Importantly, these substances retain antiviral activity in the presence of semen ${ }^{33}$. As well as inhibiting infection by free virus particles, they can block transmission by HIV-infected lymphoid cells ${ }^{43}$ (A. Profy, personal communication), and one of them - PRO 2000 - has been shown in vitro to block the attachment of HIV to DC-SIGN (R. Shattock, personal communication). They also show activity against other STI organisms, including the genital herpes virus, N. gonorrhoeae, C. trachomatis and Trichomonas vaginalis ${ }^{44,45}$.

The relatively high molecular masses of these substances mean that they are poorly absorbed through the vaginal epithelium, reducing the risk of systemic toxicity. Several anionic polymers have been shown to be protective, to varying degrees, against vaginal challenge with 


\section{a Nonoxynol-9}

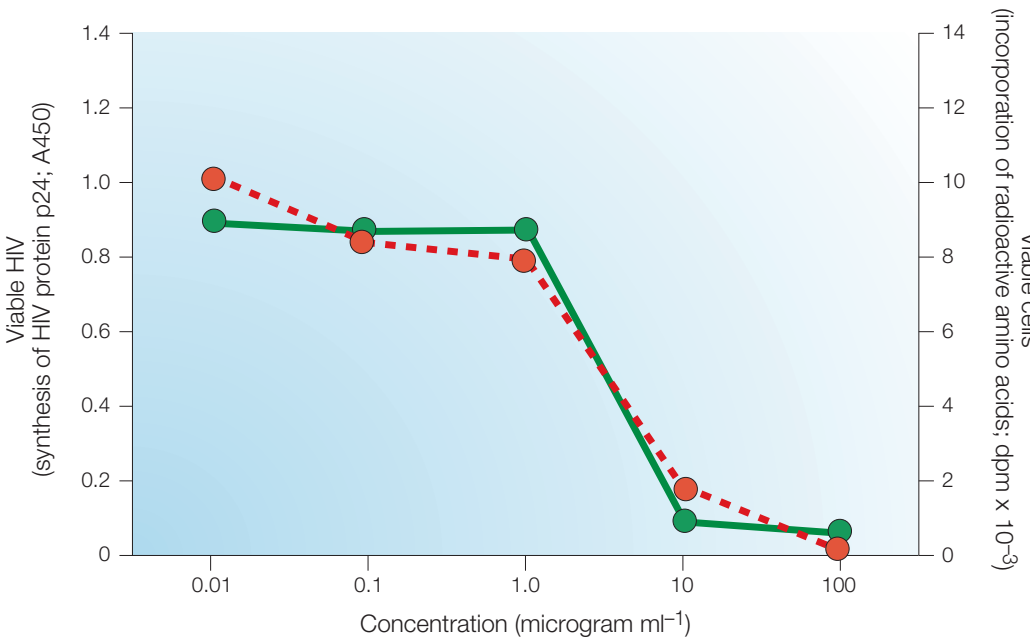

b PRO 2000

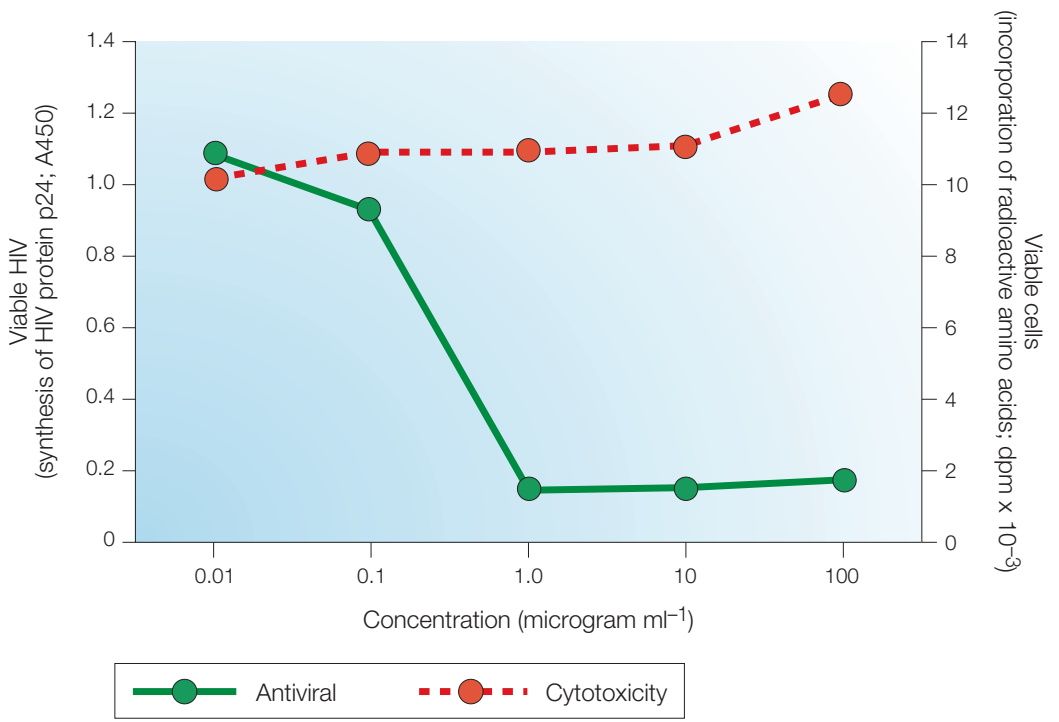

Figure 3 | Comparison of nonoxynol-9 with the anionic polymer PRO 2000: anti-HIV potency and cytotoxicity in vitro. a | Nonoxynol-9 inactivates both the virus and the cells. b | By contrast, PRO 2000 affects only the virus. A450, optical absorbance at wavelength $450 \mathrm{~nm}$; dpm, disintegrations per minute.

TERIC HINDRANCE Interference with a molecular interaction by the spatial arrangement of the structures involved.

MONOCLONAL ANTIBODY Multiple copies of a single antibody derived from the identical descendants of a single antibody-producing cell.
SIV or SHIV in the macaque. These include dextrin-2sulphate ${ }^{33}$, PRO 2000 (REFS 33,34), cellulose acetate phthalate ${ }^{35}$ and carrageenan (J. van de Wijgert, personal communication). PRO 2000 (REF. 39), carrageenan ${ }^{40}$ and cellulose acetate phthalate ${ }^{41}$ have also been shown to protect against genital herpes virus in the mouse model.

Anionic polymers do not physically destroy HIV, as do the surfactants, but work by preventing the attachment-fusion step of the infection process. Exactly how they do this is unclear (FIG.4). It is possible that as a result of their high charge, they act as 'sticky' molecules that interfere relatively non-specifically with the virus-cell interaction through STERIC HINDRANCE ${ }^{46}$. At the same time, there is some evidence for a more specific mechanism by which the polymer binds to the particular gp120 domain that interacts with the co-receptor ${ }^{47,48}$.
The HIV strains involved in human sexual transmission generally show a preference for the CCR5 coreceptor (R5 strains), whereas the SHIV strains used in many of the macaque experiments have a preference for the CXCR4 co-receptor (X4 strains) and are therefore less biologically relevant ${ }^{49}$. Studies in vitro have shown that anionic polymers are equally effective in blocking infection by either type of HIV if the microbicide is present when the virus interacts with its host cells. However, direct inactivation of virus particles before their interaction with the cells requires a higher concentration for R5 than for R4 viruses (R. Shattock, personal communication), and this might be relevant to microbicide activity against HIV in the vaginal lumen.

Several anionic polymers, including carrageenan, dextrin-2-sulphate, PRO 2000, cellulose sulphate and polystyrene sulphonate, have successfully undergone clinical studies at the Phase I level or beyond, providing evidence of their safety and their acceptability to the individuals who participated ${ }^{50-53}$.

Lowering the vaginal $p H$. Certain microbicides are designed to enhance the natural defences of the vagina. BufferGel, an acidifying agent that is intended to reduce the vaginal $\mathrm{pH}$ and keep it low even after the arrival of the alkaline ejaculate, has completed Phase I trials successfully ${ }^{54}$. A Lactobacillus suppository, designed to re-colonize the vagina with these lacticacid- and hydrogen-peroxide-producing bacteria, has also completed Phase I trials (S. Hillier, unpublished observations).

Microbicides at earlier stages of development. Several other agents are still in the process of early clinical testing, and far more are undergoing preclinical investigation. Cyanovirin is a potent HIV-inactivating protein that has low cytotoxicity. It was originally isolated from a blue-green alga but is now produced by recombinant technology. It binds to gp120 and prevents its interaction with CD4 and co-receptors on the host cell ${ }^{55}$. The antibiotic microbicides include naturally occurring cyclic peptides, such as the defensins $s^{56}$ and gramicidin ${ }^{33}$. These act as ionophores, intercalating into the lipid membrane of the pathogen and thereby creating pores (FIG. lb). The use of specific antibodies as microbicides has become a realistic option now that humanized MONOCLONAL ANTIBODIES can be produced in large quantities in plants into which the relevant genes have been engineered ${ }^{57}$. It has been shown that these 'plantibodies' can remain intact and active for many hours in the human vaginal environment.

Some of the antiretroviral drugs originally developed for therapeutic use - for example, reverse-transcriptase inhibitors, such as tenofovir - are now being evaluated as microbicides (FIG. 5). The reverse-transcriptase enzyme of HIV, which exists within the virus particle, is responsible for a crucial early stage of HIV replication in its host cell: the synthesis of a DNA copy of the viral RNA. These drugs block that step. Of course, they will not prevent migratory dendritic cells from transporting non-replicating HIV particles bound to DC-SIGN. 
a Non-specific?
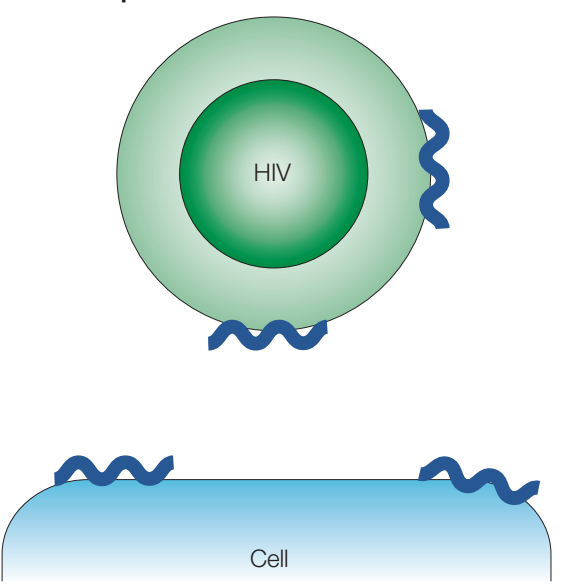

$\sim$

Anionic polymer b Specific?

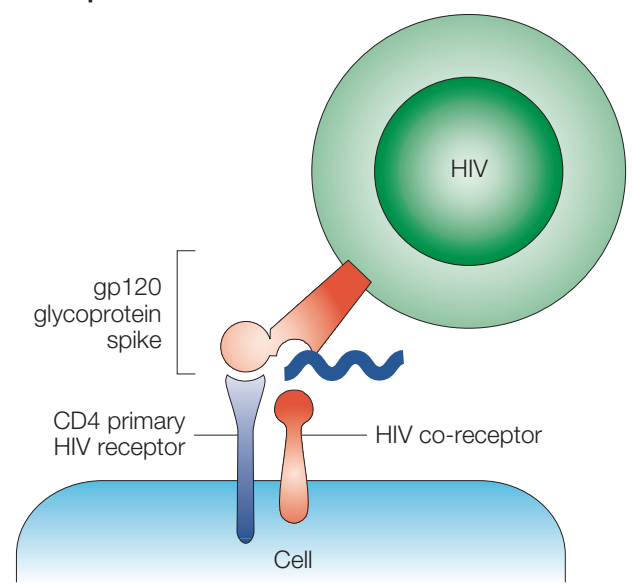

Figure 4 | Anionic polymers as microbicides: mechanism of action. The precise mechanism is unclear. a | The charged polymer might bind non-specifically to the viral and cell surfaces and prevent the close encounter required for infection to take place. $\mathbf{b}$ | The polymer might block the interaction between a specific gp120 domain and the HIV co-receptor on the cell surface. (The diagram shows only one of numerous gp120 'spikes' on the lipid envelope of HIV.)

Fortunately, however, some drugs, such as UC781, have such a high affinity for the reverse transcriptase that they bind to it inside the HIV particle, even before the virus enters the cell, and therefore render it noninfectious ${ }^{58}$. Issues that remain to be resolved include the potential for long-term toxicity if these drugs are absorbed into the systemic circulation to any significant extent, and the possible emergence of drug-resistant HIV strains. Although cost might be another issue, this should decrease as production rates grow.

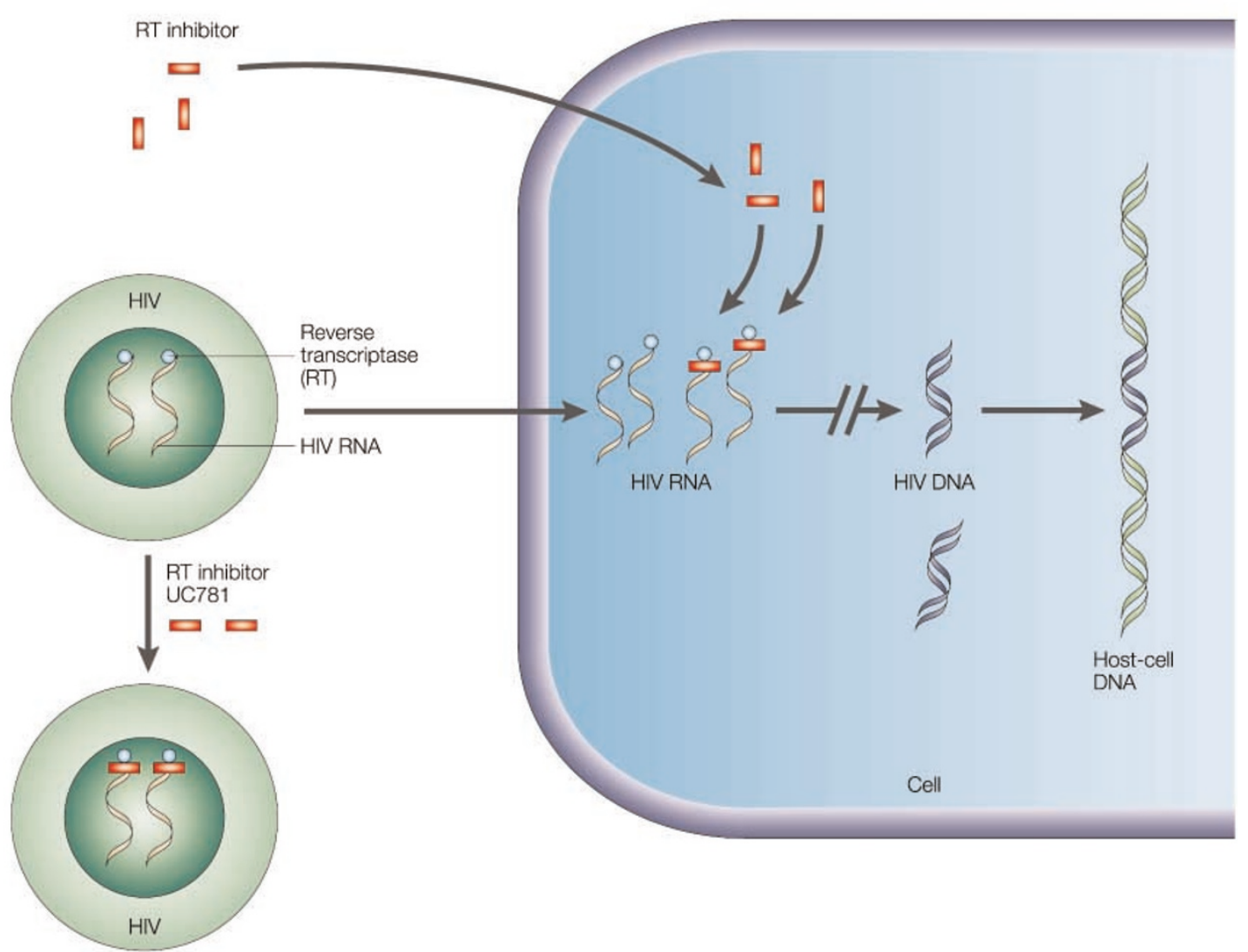

Figure 5 | Antiretroviral drugs (reverse-transcriptase inhibitors) as microbicides. These drugs enter the cell and lie in wait for any HIV genomes that manage to get in. Some of them, such as UC781, can penetrate the intact HIV particle and disable its reverse-transcription mechanism even before the virus infects a cell. 
Microbicide combinations. The notion of combining two or more microbicides that have different mechanisms of action within a single product is an attractive one, and more work on this is highly desirable. Combination products could in principle provide a greater degree of protection than single agents, a broader spectrum of activity against various pathogens and, by allowing a lower dose of each component, a lower risk of adverse reactions.

Product formulation. In parallel with research on the active agents, attention is being given to the best ways of formulating products, and to the design of suitable applicators, so that microbicides are easy to use, well tolerated, appropriately distributed in the vagina and retained there for several hours. Distribution and retention can be investigated by magnetic-resonance imaging techniques and this has shown that gels can be retained in the vagina for many hours, even in ambulatory women $^{59}$ (C. Lacey, personal communication). The possibility of providing microbicides through sustainedrelease systems, such as impregnated silicone intravaginal rings or loaded cervical caps, is also being explored.

Rectal microbicides. Efforts are also being made to develop microbicides for rectal use, to combat HIV infection as a result of anal sex. This presents some difficult challenges. The rectum is an open-ended system, not a pouch like the vagina, so achieving a stable and adequate distribution of product will be problematic. Ensuring product safety will also be challenging. The epithelium that lines the rectum is not a tough, multilayered structure like the vaginal epithelium, but a single layer of columnar cells. This is easily damaged and offers little protection to the underlying lamina propria, which is rich in $\mathrm{CD}^{+}$macrophages and dendritic cells that are readily infectable by HIV. Nonoxynol- 9 products have often been used in rectal sex. However, there are now active campaigns to ban the use of this surfactant in sex lubricants and condoms. It has been shown to enhance rectal infection by genital herpes virus in mice ${ }^{60}$ and its injurious effect on the human rectal epithelium is pronounced ${ }^{61}$.

\section{The future}

Several microbicides are scheduled to enter Phase III clinical trials over the next year or so, including carrageenan, BufferGel, PRO 2000 and dextrin-2-sulphate. Others will follow. The report of a recent international consultation sponsored by the Rockefeller Foundation ${ }^{62}$ concluded that at least one microbicide could be on the market as early as 2007. These first-generation microbicides might be no more than $60 \%$ effective against HIV transmission, but they would meet the urgent need for this kind of intervention and would have a major public health benefit. The results of epidemiological modelling indicate that such a microbicide could avert 3.7 million infections globally over a three-year period if it were used in only $50 \%$ of sex acts not protected by condoms, and assuming that it was used by only $30 \%$ of individuals easily reached by existing health services ${ }^{63}$. Concern has sometimes been expressed about the possibility that the introduction of such a product could lead to migration away from condom use and thereby increase overall HIV risk. Mathematical modelling indicates that although this might be a problem if there is a high level of condom use, say in more than $70 \%$ of sex acts, if condoms are used fairly infrequently, say in $30 \%$ of sex acts, a considerable level of migration to microbicide could be tolerated without increasing HIV risk ${ }^{64}$.

By 2012, second-generation products could be on the market ${ }^{62}$. These might be $70-90 \%$ effective against HIV, with good activity against gonorrhoea, chlamydia, genital herpes and papilloma virus. The Rockefeller report further predicted that by 2017 , microbicides would be $85-97 \%$ effective against HIV, and a 90-97\% contraceptive option would be available.

In reality, how soon these benefits materialize will depend on how successful we are in tackling not only the scientific challenges but also several societal issues. The level of funding for microbicide development needs to be greatly increased. The Rockefeller consultation estimated the requirement over the next five years to be US $\$ 775$ million, whereas the overall level of public support for the field over that period stood at approximately US $\$ 230$ million $^{62}$. This can be compared with an estimated US \$400 million per annum of public support for AIDS vaccine development ${ }^{65}$. Microbicide advocacy needs to be intensified so that health policy officials, health providers and potential end-users are properly informed. Knowledge and expertise must be shared internationally. Finally, there is growing evidence that in the longer term, microbicides could be highly profitable ${ }^{8,9,62}$, and the current reluctance of the major pharmaceutical companies to engage seriously in this field needs to be overcome. The ultimate objective is to provide a user-friendly option that sexually active people might choose to use to protect themselves against HIV, other sexually transmitted diseases and unwanted pregnancy. Microbicides will not solve the immense problem of HIV and AIDS alone, but together with condoms, and eventually AIDS vaccines, they will give people a wider choice of protective technologies.
1. Hicks, D. et al. Inactivation of HTLV-III/LAV-infected cultures of normal human lymphocytes by nonoxynol-9 in vitro. Lancet 2, 1422-1423 (1985).

2. Malkovsky, M., Newell, A. \& Dalgleish, A. Inactivation of HIV by nonoxynol-9. Lancet 1, 645 (1988).

3. Saracco, A et al. Man-to-woman sexual transmission of HIV: Iongitudinal study of 343 steady partners of infected men. J. Acquir. Immune Defic. Syndr. 6, 497-502 (1993). de Vincenzi, I. A longitudinal study of human immunodeficiency virus transmission by heterosexual partners. N. Engl. J. Med. 331, 341-346 (1994).
5. Joint United Nations Programme on HIV/AIDS and World Health Organization. AIDS Epidemic Update: December 2001 (Joint United Nations Programme on HIV/AIDS and World Health Organization, Geneva, 2001).

6. Gerbase, A. Rowley, J., Heymann, D. Berkley, S. \& Piot, P. Global prevalence and incidence estimates of selected Global prevalence and incidence estimates of selected curable STDs. Sex. Transm. Infect. 74 (Suppl. 1), S12-S16 (1998).

7. Coggins, C., Blanchard, K. \& Friedland, B. Men's attitudes towards a potential vaginal microbicide in Zimbabwe, Mexico and the USA. Reprod. Health Matters 8, 132-141 (2000).
8. Darroch, J. \& Frost, J. Women's interest in vaginal microbicides. Fam. Plann. Perspect. 31, 16-23 (1999).

9. Hill, R Ryan, J Stone, A. \& Fransen, L Vaginal microbicides for the prevention of HIV/AIDS: a bicides for the prevention of HIVIAIDS. assessment of the

0. Kotential J er a. Eft. J. Pham Med 14, 271-278 (2000). sponge use in preventing heterosexual acquisition of HIV in Nairobi prostitutes. JAMA 268, 477-482 (1992).

11. Rosenberg, Z., Van Damme, L. \& Mauck, C. Recommendations for the clinical development of topical microbicides: an update. AIDS 15, 857-868 (2001). 
12. Roddy, R. et al. A controlled trial of nonoxynol-9 film to reduce male-to-female transmission of sexually transmitted diseases. N. Engl. J. Med. 339, 504-510 (1998)

13. Van Damme, L. et al. Effectiveness of COL-1492, a nonoxynol-9 vaginal gel, on HIV-1 transmission in female sex workers: a randomised controlled trial. Lancet $\mathbf{3 6 0}$, 971-977 (2002)

This large-scale (Phase III) clinical trial of nonoxynol-9 gel helped to drive home the message that products that contain this surfactant are not only ineffective in protecting against HIV and other STIs, but can actually increase HIV risk, probably by causing ulcers in the genital epithelium.

14. Van Damme, L. et al. Safety of multiple daily applications of COL-1492, a nonoxynol-9 vaginal gel, among female sex workers. AIDS 14, 85-88 (2000).

15. Roddy, R., Zekeng, L., Ryan, K., Tamoufe, U. \& Tweedy, K. Effect of nonoxynol-9 on urogenital gonorrhoea and chlamydial infection. JAMA 287, 1117-1122 (2002).

16. World Health Organization/Contraceptive Research and Development Program. WHO/CONRAD Technical Consultation on Nonoxynol-9 (World Health Organization, Geneva, 2002).

A report of an expert consultation that took place in October 2001 to consider the available evidence on the efficacy and toxicity of nonoxynol-9, both as a microbicide and as a spermicidal contraceptive. The report concluded that nonoxynol-9 should not be used for HIV/STI prevention, and that there is an urgent need to develop safe and effective microbicides. It also recommended that nonoxynol-9 spermicides, although inefficient contraceptives, should be retained as a female-controlled contraceptive option provided that HIV risk is low.

7. Brelot, A. \& Alizon, M. HIV-1 entry and how to block it. AIDS 15 (Suppl. 5), S3-S11 (2001).

18. Greenhead, P. et al. Parameters of human immunodeficiency virus infection of human cervical tissue and inhibition by vaginal virucides. J. Virol. 74, 5577-5586 (2000). Explants of human vaginal and cervical epithelium maintained in culture fluid are used to study their infectabilty by different HIV strains, the effects of immune activation and the locations and types of cell initially infected. Several microbicides are shown to be active in preventing infection in this system.

19. Spira, A. et al. Cellular targets of infection and route of vira dissemination after an intravaginal inoculation of simian immunodeficiency virus into rhesus macaques. $J$. Exp. Med. 183, 215-225 (1996).

20. Zhang, L. et al. In vivo distribution of human immunodeficiency virus/simian immunodeficiency virus coreceptors: CXCR4, CCR3 and CCR5. J. Virol. $\mathbf{7 2}$, 5035-5045 (1998).

21. Geijtenbeek, T. et al. DC-SIGN, a dendritic cell-specific HIV-1-binding protein that enhances transinfection of T-cells. Cell 100, 587-597 (2000)

This paper describes a potential new route of HIV sexual transmission. HIV particles can bind to a specific lectin, DC-SIGN, which is present in the specific lectin, DC-SIGN, which is present in the
membrane of human genital dendritic cells. HIV membrane of human genital dendritic cells. HIV particles so bound retain their infectivity for sever
days and can be efficiently transmitted to $T$ cells.

22. Bomsel, M. Transcytosis of infectious human immunodeficiency virus across a tight human epithelial cell line barrier. Nature Med. 3, 42-47 (1997)

23. Collins, K., Patterson, B., Naus, G., Landers, D. \& Gupta, P. Development of a new in vitro organ culture model to study transmission of HIV-1 in the female genital tract. Nature Med. 6, 475-479 (2000).

24. Shattock, R., Griffin, G. \& Gorodeski, G. In vitro models of mucosal HIV transmission. Nature Med. 6, 607-608 (2000).

25. Gupta, P., Collins, K., Patterson, B., Naus, G. \& Landers, D. In vitro models of mucosal HIV transmission. Nature Med. 6, 607-608 (2000).

26. Stone, A. Microbicides: the UNAIDS-sponsored trial of nonoxynol-9 gel. IPPF Med. Bull. 34, 3-4 (2000).

27. Stafford, M. et al. Safety study of nonoxynol-9 as a vaginal microbicide: evidence of adverse effects. J. Acquir. Immune Defic. Syndr. 17, 327-331 (1998)

28. Klebanoff, S., Hillier, S., Eschenbach, D. \& Waltersdorph, A. Control of the microbial flora of the vagina by $\mathrm{H}_{2} \mathrm{O}_{2}-$ generating lactobacilli. J. Infect. Dis. 164, 94-100 (1991).

29. Ongradi, J., Ceccherini-Nelli, L., Pistell, M., Spector, S. \& Bendinelli, M. Acid-sensitivity of cell-free and cellassociated HIV-1: clinical implications. AIDS Res. Hum. Retroviruses 6, 1433-1436 (1990).

30. Wasserheit, J. in AIDS and Women's Health: Science for Policy and Action (eds Chen, L., Sepulveda, J. \& Segal, S.) 47-72 (Plenum, New York, 1991).
31. O'Connor, T., Kinchington, D., Kangro, H. \& Jeffries, D. The activity of candidate virucidal agents, low pH and genital secretions against HIV-1 in vitro. Int. J. STD AIDS 6, 267-272 (1995)

32. Miller, C., Alexander, N., Gettie, A., Hendrickx, A. \& Marx, P. The effect of contraceptives containing nonoxynol-9 on the genital transmission of simian immunodeficiency virus in rhesus macaques. Fertil. Steril. 57, 1126-1129 (1992).

33. Weber, J. et al. 'Chemical condoms' for the prevention of HIV infection: evaluation of novel agents against SHIV (89.6PD) in vitro and in vivo. AIDS 15, 1563-1568 (2001).

34. Lewis, M. et al. Efficacy of PRO 2000 gel in a macaque model for vaginal HIV transmission. 8th Conference on Retroviruses and Opportunistic Infections Abstract 721 (2001).

35. Manson, K., Wyand, M., Miller, C. \& Neurath, R. Effect of cellulose acetate phthalate topical cream on vaginal transmission of simian immunodeficiency virus in rhesus monkeys. Antimicrob. Agents Chemother. 44, 3199-3202 (2000).

36. Tevi-Benissan, C. et al. Protection of cynomolgus macaque against cervicovaginal transmission of SIVmac251 by the spermicide benzalkonium chloride. J. Acquir. Immune Defic. Syndr. 24, 147-153 (2000).

37. Di Fabio, S. et al. Vaginal transmission of HIV-1 in hu-SCID mice: a new model for the evaluation of vaginal microbicides. AIDS 15, 2231-2238 (2001).

38. Kish, T., Budgeon, L., Welsh, P. \& Howett, M. Immunological characterisation of human vaginal xenografts in immunocompromised mice: development of a small animal model for the study of human immunodeficiency virus-1 infection. Am. J. Pathol. 159 2331-2345 (2001)

39. Bourne, N. et al. The topical microbicide PRO 2000 protects against genital herpes infection in a mouse model. J. Infect. Dis. 180, 203-205 (1999)

40. Zacharopoulos, V. \& Phillips, D. Vaginal formulations of carrageenan protect mice from herpes simplex virus infection. Clin. Diagn. Lab. Immunol. 4, 465-468 (1997).

41. Gyotoku, T., Aurelian, L. \& Neurath, R. Cellulose acetate phthalate (CAP): an 'inactive' pharmaceutical excipient with antiviral activity in the mouse model of genital herpesvirus infection. Antivir. Chem. Chemother. 10, 327-332 (1999).

42. The International Working Group on Vaginal Microbicides. Recommendations for the development of vaginal microbicides. AIDS 10, 1-6 (1996).

This was the first such compilation to be published, providing useful guidance to those involved in microbicide development. The grou has since published more comprehensive and up-to-date clinical recommendations (reference 10), and updated preclinical recommendations will appear shortly.

43. Javan, C., Gooderham, N., Edwards, R., Davies, D. \& Shaunak, S. Anti-HIV type 1 activity of sulphated derivatives of dextrin against primary viral isolates of HIV type 1 in Iymphocytes and monocyte-derived macrophages. AIDS Res. Hum. Retroviruses 13, 875-880 (1997).

44. Profy, A. et al. PRO 2000 gel, a potential topical microbicide for HIV prevention, can block infection by other sexually transmitted disease pathogens. $X$ International AIDS Conference Abstract 33146 (1998).

45. Neurath, R., Strick, N., Li, Y.-Y., Lin, K. \& Jiang, S. Design of a 'microbicide' for prevention of sexually transmitted diseases using 'inactive' pharmaceutical excipients. Biologicals 27, 11-21 (1999).

46. McClure, M. et al. Investigations into the mechanism by which sulphated polysaccharides inhibit HIV infection in which sulphated polysaccharides inhibit HIV infection

47. Neurath, A., Strick, N., Li, Y.-Y. \& Debnath, A. Cellulose acetate phthalate, a common pharmaceutical excipient, inactivates HIV-1 and blocks the coreceptor binding site on the virus envelope glycoprotein gp120. BMC Infect. Dis. 1, 17 (2001).

48. Moulard, M. et al. Selective interactions of polyanions with basic surfaces on human immunodeficiency virus type 1 gp120. J. Virol. 74, 1948-1960 (2000).

49. Shattock, R. \& Doms, R. AIDS models: microbicides could learn from vaccines. Nature Med. 8, 425 (2002).

50. Coggins, C. et al. Preliminary safety and acceptability of a carrageenan gel for possible use as a vaginal microbicide. Sex. Transm. Infect. 76, 480-483 (2000).

51. Stafford, M. et al. A placebo-controlled, double-blind prospective study in healthy female volunteers of dextrin sulphate gel: a novel potential intravaginal virucide. J. Acquir. Immune Defic. Syndr. 14, 213-218 (1997).

52. Van Damme, L. et al. A Phase I study of a novel potential intravaginal microbicide, PRO 2000, in healthy sexually inactive women. Sex. Transm. Infect. 76, 126-130 (2000).
53. Mauck, C. et al. Single and multiple exposure tolerance study of cellulose sulfate gel: a Phase I safety and colposcopy study. Contraception 64, 383-391 (2001).

54. Mayer, K. et al. Safety and tolerability of BufferGel, a novel vaginal microbicide, in women in the United States. Clin. Infect. Dis. 32, 476-482 (2001)

55. Dey, B. et al. Multiple antiviral activities of cyanovirin-N: blocking of gp120 interaction with CD4 and coreceptor, and inhibition of diverse enveloped viruses. J. Virol. $\mathbf{7 4}$ 4562-4569 (2000).

56. Cole, A. et al. Retrocyclins: circular minidefensins with potent activity against HIV-1. Microbicides 2002 Conference Abstract A-048 (2002).

57. Zeitlin, L. et al. A humanized monoclonal antibody produced in transgenic plants for immunoprotection of the vagina against genital herpes. Nature Biotechnol. 16, 1361-1364 (1998)

58. Balzarini, J. et al. Preclinical studies on thiocarboxanilide UC-781 as a virucidal agent. AIDS 12, 1129-1138 (1998).

59. Barnhart, K. T., Pretorius, E. S. \& Malamud, D. Non-invasive imaging of the spread of microbicides with magnetic resonance imaging. Microbicides 2002 Conference Abstract B/C-283 (2002).

60. Phillips, D. \& Zacharopoulos, V. Nonoxynol-9 enhances rectal infection by herpes simplex virus in mice. Contraception 57, 341-348 (1998).

61. Phillips, D., Taylor, C., Zacharopoulos, V. \& Maguire, R. Nonoxynol-9 causes rapid exfoliation of sheets of rectal epithelium. Contraception 62, 149-154 (2000). This study showed that two over-the-counte nonoxynol- 9 products caused considerable damage to the human rectal epithelium within 15 minutes of being applied by volunteers. This finding, together with the material in references 13 and 16 , have led to calls for a ban on condoms coated with nonoxynol-9 and sex lubricants containing this substance.

62. Rockefeller Foundation. Mobilization for Microbicides: the Decisive Decade (Rockefeller Foundation, New York, 2002).

63. Watts, C. The public health impact of microbicides: mode projections. Microbicides 2002 Conference [online] (cited 15 Oct 2002) <http://www.itg.be/micro2002/pages/ presentations.html> (2002).

An influential study that used epidemiological modelling techniques to show that a microbicide of only moderate efficacy could have substantial public health benefits in terms of HIV infections averted, provided that, as a female-controlled method, it is used more consistently than condoms.

64. Foss, A., Vickerman, P., Heise, L. \& Watts, C. Will shifts from condom to microbicide use increase HIV risk? Mode projections. XIV International AIDS Conference Abstract WeOrD1319 (2002)

65. International AIDS Vaccine Initiative. When Will an AIDS Vaccine be Found? The State of Global Research (International AIDS Vaccine Initiative, New York, 2002).

Acknowledgements

The author is grateful to A. Profy and D. Jeffries for allowing the inclusion of figure 3 , the data for which were derived by the late T. O'Connor in D. Jeffries' laboratory. Thanks also to R. Shattock for valuable discussions concerning the mechanism of HIV infection. A. S. is Consultant to International Family Health. $\mathrm{He}$ is a member of the Management Board of the Medical Research Council (MRC)/Department for International Development (DFID) Microbicides Development Programme and Chairman of the International Working Group on Microbicides. This article is a contribution to the Microbicides Advocacy and Networking Project supported by a European Commission award to International Family Health. The views expressed are those of the author and do not necessarily reflect the positions of the above organisations.

\section{(2) Online links}

\section{DATABASES}

The following terms in this article are linked online to: LocusLink: http://www.ncbi.nlm.nih.gov/LocusLink/

CCR5 | CD4 | CXCR4

\section{FURTHER INFORMATION}

Alliance for Microbicide Deve

Encyclopedia of Life Sciences: http://www.els.net/ acquired immune deficiency syndrome | AIDS as a world health problem | human immunodeficiency viruses Global Campaign for Microbicides: http://www.global-campaign.org/ International AIDS Vaccine Initiative: http://www.iavi.org/ International Family Health: http://www.ifh.org.uk/ Rockefeller Foundation: http://www.rockfound.org/ World Health Organization: http://www.who.int/en Access to this interactive links box is free online. 